\title{
El microcrédito como potenciador del desarrollo local: Análisis de las condiciones de vida
}

\author{
Microcredit as a stimulant of local development: Analysis of living conditions
}

\section{Edison Duta-Uyaguary ${ }^{*}{ }^{*}$, Gabriela Álava-Atiencie ${ }^{2}$ iD, Sonia Sigüenza-Orellana ${ }^{3}$ (D), Lucía Pinos-Ramón ${ }^{4}$}

${ }^{1}$ Egresado de la Maestría en Desarrollo Local, Facultad de Ciencias Económicas y Administrativas, Universidad de Cuenca. Cuenca, Ecuador.

${ }^{2}$ Docente Investigadora, Facultad de Ciencias Económicas y Administrativas, Universidad de Cuenca. Cuenca, Ecuador.

${ }^{3}$ Investigadora, Programa de Acompañamiento Organizacional al Desarrollo (ACORDES), Facultad de Ciencias Económicas y Administrativas, Universidad de Cuenca. Cuenca, Ecuador.

${ }^{4}$ Investigadora, Programa de Acompañamiento Organizacional al Desarrollo (ACORDES), Facultad de Ciencias Económicas y Administrativas, Universidad de Cuenca. Cuenca, Ecuador.

* Autor de correspondencia: emduagosto@ hotmail.com

Fecha de recepción: 2 de noviembre de 2021 - Fecha de aceptación: 17 de diciembre de 2021

\section{RESUMEN}

Esta investigación estudia el microcrédito como potenciador del desarrollo local mediante el análisis de su impacto en las condiciones de vida de los miembros de la Cooperativa de Ahorro y Crédito Riobamba Ltda. El análisis se basa en una encuesta aplicada a una muestra de 548 personas, que fueron seleccionadas en función de la zona de residencia, urbana versus rural y género. El propósito de la encuesta fue determinar cómo las personas encuestadas perciben el impacto de los microcréditos en sus condiciones de vida. Para el procesamiento de la información recolectada se utilizó una técnica cuantitativa descriptivaexplicativa y correlacional. Los resultados mostraron que la mayoría de los miembros urbanos creen que el microcrédito les permitió mejorar sus condiciones de vida personales, familiares, microempresariales y comunitarias, mientras que la mayoría de los miembros rurales perciben mejor impacto a nivel microempresarial. Además, se evidencia que hay una proporción de asociados para quienes el microcrédito ha significado un deterioro en sus condiciones de vida tanto a nivel personal, familiar, microempresarial y comunitario, siendo mayor esta percepción en zonas urbanas. Las diferencias en las puntuaciones medias de la percepción de impacto del microcrédito a nivel personal, familiar, microempresarial y comunitario por zonas son estadísticamente significativas. En conclusión, el microcrédito como alternativa financiera es un instrumento ideal para mejorar las condiciones de vida de los miembros y sus familias de la COAC Riobamba, lo que se traduce en mejores condiciones de vida a nivel comunitario.

$\underline{\text { Palabras clave: }}$ Desarrollo Local, servicios financieros, cooperativa, microcrédito, condiciones de vida.

\begin{abstract}
This research studies microcredit as an enhancer of local development by analyzing its impact on the living conditions of the members of the Cooperativa de Ahorro y Crédito Riobamba Ltda. The analysis is based on a survey applied to a sample of 548 people, who were selected according to area of residence, urban versus rural, and gender. The purpose of the survey was to determine how respondents perceive the impact of microcredit on their living conditions. A quantitative descriptiveexplanatory and correlational descriptive-explanatory technique was used to process the information collected. The results showed that most of the urban members believe that microcredit allowed them to improve their personal, family, microenterprise and community living conditions, while most of the rural members perceive better impact at the microenterprise level. In addition, it is evident that there is a proportion of members for whom the microcredit has meant a deterioration in their personal, family, microenterprise and community living conditions, with this perception being greater in urban areas. The differences in the mean scores of the perception of the impact of microcredit at the personal, family, microenterprise and community levels by zones are statistically significant. In conclusion, microcredit as a financial alternative is an ideal instrument to improve the living conditions of the members and their families of COAC Riobamba, which translates into better living conditions at the community level.
\end{abstract}

Keywords: Local development, financial services, cooperatives, microcredit, living conditions. 
1.

\section{INTRODUCCIÓN}

La economía mundial presenta una creciente disparidad entre los ricos y los pobres. Donde los primeros disfrutan de todas las comodidades y privilegios, en tanto, que los segundos viven privaciones que limita su acceso a una alimentación sana, educación, salud, vivienda. Sumado a lo dicho, las personas pobres se enfrentan a la imposibilidad de acceder a fuentes de financiamiento que les permita generar oportunidades de trabajo autónomo. En este contexto, el microcrédito conceptualizado como "programas de concesión de pequeños créditos a los más necesitados de entre los pobres para que estos puedan poner en marcha pequeños negocios que generen ingresos para mejorar su nivel de vida y el de sus familias" (Naciones Unidas, 1997), se constituye en una oportunidad para generar autoempleo.

El microcrédito tiene sus orígenes en los años setenta en Bangladesh, cuando, el economista Mohammad Yunus, premio Nobel de la Paz en 2006, fundó, el Banco Grameen de Blangladesh conocido también como "Banco para pobres" a través del cual, se promovió el microcrédito dirigido a las familias de escasos recursos, con la finalidad de mejorar las condiciones de vida e incentivar la implementación o fortalecer emprendimientos como medios de sustentos de estas familias (Carvajal-Salgado \& Espinoza-Párraga, 2020). El microcrédito como operación crediticia para el sector microempresarial, inicia en el año 1986, desde un enfoque de "alivio a la pobreza", es decir, acompañado de capacitación y asistencia técnica. Sin embargo, su impacto fue reducido y limitado. Ya, en el año 2002, mediante Resolución 457 se cuenta con la primera normativa que regula y controla a las microfinanzas, situación que permitió que el mercado se vuelva dinámico y los microcréditos más acogidos.

De manera general, desde sus inicios el microcrédito fue creado para financiar y aportar al desarrollo económico y social de la población que no dispone de fuente fija de ingresos o estos son bajos que no constituyen una garantía suficiente para los mercados financieros tradicionales (García, 2011). De manera especial, a las mujeres, quienes son las más pobres entres las personas pobres. Para Cairó I Céspedes \& Gómez-Gonzáles (2015), el microcrédito se constituye en una herramienta para alcanzar la igualdad de oportunidades y el empoderamiento económico, social y político de las mujeres. Por su parte Sen (2000), premio Nobel de Economía en 1998, afirma que el microcrédito es un elemento esencial para generar autoempleo en las familias, aportando de esta manera, al mejoramiento de sus condiciones de vida. Es decir, el acceso a crédito se constituye en factor esencial que permite a quienes están excluidos del sistema financiero formal, ya sea por no contar con avales o garantías requeridas, tener acceso a una fuente de financiamiento en mejores condiciones que las que ofrece el mercado informal. Esta situación aporta al fortalecimiento del autoempleo y permite percibir mayores ingresos, por ende, experimentan un mejoramiento en sus condiciones de vida en aspectos relacionados con: educación, vivienda, salud, alimentación (Rué Cabré, 2006; Martínez-Centanaro, 2017).

Para Cairó I Céspedes \& Gómez González (2015) el microcrédito puede ser entendido desde dos enfoques: "autosuficiencia financiera" y "alivio de la pobreza". Desde el primer enfoque mencionado, se afirma que el microcrédito está dirigido a los "menos pobres entre los pobres", se considera que estos por sí solo permiten mejorar las condiciones socioeconómicas de las personas. El segundo enfoque, coincide respecto de las personas beneficiarias, sin embargo, se considera que el microcrédito debe brindar otros servicios no financieros como facilitar espacios de encuentro y el desarrollo de capacitaciones, porque estas estrategias facilitarán el desarrollo de actividades sustentables y generadoras de excedentes económicos. Situación que aporta al mejoramiento de los ingresos y bienes, al acceso a salud y a educación y, el fortalecimiento de redes de apoyo social (pp. 37-38).

Se debe recalcar que el presente artículo se enmarca en el enfoque de alivio de la pobreza. Desde este enfoque, Carvajal-Salgado \& Espinoza-Párraga (2020) identifican algunas ventajas y desventajas del microcrédito. Entre las ventajas se nombra el acceso a recursos económicos para emprender, la seguridad de disponer de recursos con instituciones fiables y, costos financieros bajos. Entre las desventajas las autoras identifican el uso del dinero para gastos personales y no para el negocio. Al ser los montos bajos, generalmente estos no cubren la inversión necesaria para ejecutar la idea de negocio y, los períodos de pagos son cortos, situación que dificulta consolidar un capital de trabajo sólido.

En definitiva, el microcrédito como instrumento social aporta al desarrollo de las potencialidades de cada persona. Además, facilita el acceso a recursos financieros, en condiciones favorables, que permite a las personas la posibilidad de emprender, ya que reciben adicionalmente asistencia técnica y capacitación. Desde esta perspectiva, el microcrédito es un componente importante para el crecimiento económico y la generación de cambios estructurales que limiten la exclusión social y la pobreza, pues permite la generación de actividades económicas y autoempleo, que en conjunto aportan al desarrollo local. Por consiguiente, a decir de, los microcréditos se caracterizan principalmente porque su aporte a la inclusión económica y la democratización de los servicios financieros para las personas pobres (Gómez-Lavín Fernández, 2015). De ahí que, el microcrédito puede ser considerado como potenciador del desarrollo local, ya que permite alcanzar tres objetivos de este tipo de desarrollo. Primero, fortalece, potencia y desarrolla el entramado productivo de un territorio con la participación de sus propios actores (eficiencia). Segundo, permite la inclusión de emprendedores tradicionalmente excluidos de los procesos productivos locales, por la escasa o nula posesión de bienes y garantías para acceder a los sistemas financieros tradicionales, limitando su acceso a un capital necesario que les permitiese poner en marcha sus ideas (equidad). Y, tercero, al dirigir la inversión del microcrédito a iniciativas eficientes responsables y respetuosas con el medioambiente, se estaría garantizando un desarrollo ambiental sostenible (sostenibilidad) (Sanhueza, 2019).

Hasta el momento se ha revisado la conceptualización del microcrédito, sus enfoques, características, ventajas y desventajas, no obstante, se vuelve imprescindible analizar los impactos del microcrédito como un proceso de estudio que busca evidenciar los cambios que han experimentado quienes han accedido a un microcrédito. Es decir, la evaluación de impacto del microcrédito tiene por objetivo "probar de forma fehaciente, que si la persona no hubiese obtenido ese microcrédito (variable 
independiente $\mathrm{X}$ ), los cambios en las variables dependientes $\mathrm{Y}$, no se habrían producido o se habrían producido en menor grado" (Gómez-Acebo, 2018). Los resultados deben constituirse en evidencia empírica para poder tomar decisiones.

Vallejo-Ramírez, Ochoa-Herrera, Padilla-Andrade, \& Guamán-Paladines (2018) expresan que para evaluar los impactos se debe considerar, en primer lugar, evaluar los cambios a nivel personal y familiar y, en segundo lugar, los cambios que se genera una vez que se accede al microcrédito, es decir, evaluar el impacto del microcrédito implica un proceso de estudio que evidencian los cambios económicos, sociales, educativos, psicológicos, entre otros que este genere, ya sea a nivel personal, familiar o comunitarios. Desde esta perspectiva, la Universitat de les Illes Balears (2006), expresa que para medir el impacto del microcrédito se debe definir qué tipo de impacto se desea conocer, identificando el impacto económico, para lo cual, se considera el ingreso familiar, el empleo y la adquisición de máquinas y herramientas. El impacto social y a nivel del hogar, considerando las condiciones de la vivienda, educación y el acceso a la alimentación. El impacto a nivel personal que considera el desarrollo de la autoestima, relaciones equilibradas y la capacidad en la toma de decisiones (Cátedra Iberoamericana, 2006).

Otras evaluaciones sobre el impacto del microcrédito centran su atención en el análisis de la calidad de vida, entendida esta como "la experiencia que las personas tienen de sus propias formas y condiciones de vida" (Casas, 2004). En este sentido, las condiciones de vida se refieren "al tener", a la dimensión objetiva de la calidad de vida, recae sobre los recursos materiales. De ahí que, el microcrédito como fuente de financiación aporta al fortalecimiento del autoempleo, que permiten percibir ingresos y con ello un mejoramiento en sus condiciones de vida relacionadas principalmente con la vivienda, alimentación, educación, salud, entre otras (MartínezCentanaro, 2017). Para Cisneros-Martínez \& GarcésVenegas (2021) el microcrédito aporta al mejoramiento de las condiciones de vida, ya que permite fortalecer necesidades básicas, pues si bien el crédito otorgado no va dirigido para cubrir estas necesidades, sin embargo, les permiten mejorar la economía familiar y por ende su acceso a salud, alimentación y vivienda. Por tal razón, el microcrédito se constituye en la base económica que impulsa el crecimiento económico de los emprendimientos, mismos que son mecanismos implementados para sustentar y satisfacer las necesidades básicas. Por tanto, los microcréditos pueden constituirse en "agentes que permitan un cambio estructural y productivo" (Maldonado-Román \& Armijos-Tandazo, 2017, p. 20) pues su finalidad principal es implementar una actividad comercial, potenciar un negocio, o para optimizar los recursos. En este sentido, para RodríguezGarcés (2008) el microcrédito es visto como una alternativa de subsistencia, es utilizado para aumentar el capital de trabajo, compra materia primas e insumos a menores precios, para la adquisición de herramientas y equipos con la finalidad de aumentar la producción y por ende las ventas.

Diversos estudios han evidenciado los efectos que el microcrédito ha generado en las condiciones de vida de quienes han accedido a este servicio financiero. Así para Martínez-Centanaro (2017) en su estudio realizado en Colombia, respecto de los efectos del microcrédito en las condiciones de vida el microcrédito, la autora demuestra, mediante la aplicación de una metodología mixta, que estos tienen efectos positivos en el mejoramiento de las condiciones de vida, siendo uno de los factores de incidencia la capacitación orientada a la estructuración o reformulación de su idea de negocio. Así también, la autora identifica la generación de utilidades que les permitió la adquisición de maquinaria, equipos y herramientas propias que permite el incremento de la producción y diversificación de sus productos y, por ende, mayor disponibilidad de recursos para sus hogares. De manera general, el estudio demuestra que el microcrédito, ayuda al fortalecimiento del autoempleo y a la generación de ingresos, experimentando mejoramiento en sus condiciones de vida, en aspectos relacionados con: ingresos, alimentación, educación, salud y vivienda.

Otro estudio realizado en Ecuador, por Vallejo-Ramírez, Ochoa-Herrera, Padilla-Andrade, \& Guamán-Paladines (2018), en el año 2018, respecto al impacto del microcrédito, evidencia que este están principalmente destinados al sector de personas que trabaja sin relación de dependencia que cuentan con pequeños negocios con capitales limitados. De la aplicación de una metodología mixta para la evaluación, los resultados demuestran un nivel de impacto del microcrédito de $55 \%$ aproximadamente en las condiciones sociales y económicas, porcentaje considerado promedio de acuerdo a la mejora de su vivienda, del negocio y de educación de sus hijos e hijas. Además, las personas autores, dejan en evidencia la aplicación de la economía popular y solidaria que incluye a las personas excluidas de la banca tradicional quienes buscan sus oportunidades, al momento de fortalecer las pequeñas economías, situación que se ve reflejada en el mejoramiento de las condiciones de vida a nivel familiar pero también a nivel comunitario, ya que se disminuye el desempleo.

Por su parte el estudio realizado por Peláez-Moreno, Ochoa-Herrera, Padilla-Andrade, Granda-Pardo, \& Morocho-Pasaca (2021) denominado "Estudio comparativo del microcrédito en la Comunidad Andina: una mirada al 2020", evidencia que los microcréditos han aportado significativamente a la economía de Colombia, Ecuador, Perú y Bolivia, ya que estos han estado orientados especialmente a la población carente de oportunidades, generando en ciertos casos mejora de sus condiciones de vida. No obstante, las personas autoras enfatizan que el microcrédito en la región andina ha tenido un éxito relativo, pues si bien, dado como resultado en especial crecimiento importante de los volúmenes de microcrédito, sin embargo, se observa una creciente mercantilización del mismo, situación que pone en tela de juicio la afirmación de Yunus, para quien el microcrédito aporta a la erradicación de la pobreza.

Con todo lo expresado, se plantea como hipótesis de esta investigación que los microcréditos como fuente de financiación ha permitido la generación de autoempleo de las personas asociadas a la Cooperativa de Ahorro y Crédito Riobamba Ltda., que accedieron a un microcrédito, en la ciudad de Riobamba y, con ello, han logrado percibir mejores ingresos experimentando mejores condiciones de acceso a educación, salud, alimentación, vivienda, es decir, han logrado mejores condiciones de vida. Para comprobar la hipótesis se plantea como objetivo analizar las condiciones de vida en las que el microcrédito otorgado por la Coop. Riobamba Ltda., en el período 2012-2017 contribuyó a mejorar la situación de las personas socias por zona de residencia. 
Para ello se describen las condiciones de vida en las que el microcrédito aportó a mejorar la situación de las personas socias a: a nivel personal, familiar, microempresarial, comunitario e institucional y, se prueban si las diferencias en las condiciones de vida por zona son estadísticamente significativas.

\section{MATERIALES Y MÉTODOS}

La investigación recurre a una metodología cuantitativa. $\mathrm{Su}$ alcance es descriptivo-explicativo y correlacional, permitiendo determinar, las propiedades y características del impacto de los microcréditos y establecer sus causas (Lema, Erazo, \& Narváez, 2019). Su finalidad es de tipo transversal, puesto que se realizó en un tiempo único. La unidad de análisis son las personas socias de la cooperativa de ahorro y crédito que durante el período 2012-2017, accedieron a un microcrédito de acumulación ampliada con la finalidad de invertir en actividades económicas productivas agropecuarias, artesanales y de alimentos. El tamaño poblacional fue de 6291 personas socias. Se aplica un muestreo aleatorio estratificado con afijación proporcional, considerando zonas (urbanas y rurales) y género (femenino o masculino). Con un margen de error permisible de $8 \%$, una probabilidad máxima estandarizada de $\mathrm{p}=50 \%$ y $\mathrm{q}=50 \%$, un nivel de confianza del 95\% ( $\mathrm{z}=1.96)$. De aplicación muestral se determinó una muestra de 548 personas.

Se aplicó la encuesta elaborada en el marco de la tesis de Maestría en Desarrollo Local, cursada por Edisson Duta, quien analiza el impacto socioeconómico de los Microcréditos otorgados por la Cooperativa de Ahorro y Crédito Riobamba Ltda., en la ciudad de Riobamba, durante el periodo 2012 - 2017. La caracterización de la población de estudio, en cuanto a sexo, escolaridad, zona de residencia y estado civil se obtuvo del análisis de la sección datos generales de la encuesta, aplicando a 251 mujeres y 297 hombres, en su mayoría con una escolaridad de educación secundaria $(\mathrm{n}=25 \%$ mujeres y $\mathrm{n}=35 \%$ hombres). Pertenecientes a la zona urbana $62 \%$ ( $\mathrm{n}=33 \%$ mujeres y $\mathrm{n}=29 \%$ hombres $)$ y $38 \%$ a la zona rural ( $\mathrm{n}=13 \%$ mujeres y $\mathrm{n}=25 \%$ hombres). De estado civil solteros/a $45 \%$ ( $n=29 \%$ mujeres y $n=16 \%$ hombres).

El análisis de los datos se realizó en el programa SPSS versión 21. Se aplicó técnicas de estadística descriptiva e inferencial, como ANOVA factorial. Los resultados se muestran mediante tablas y gráficos. Para evidenciar la fiabilidad y validez de las variables componentes/latentes se realiza análisis factorial exploratorio, que tiene como principal objetivo establecer la estructura subyacente entre todas las variables observables de análisis (Méndez \& Rondón, 2012). Por otra parte, para la generación de categorías para análisis de las variables componente, de acuerdo con la sugerencia de Álava-Atiencie (2019) se recodifica las variables componentes formadas, ya que el análisis factorial genera las variables estandarizadas como coeficientes de regresión, que siguen una distribución normal, con media cero y varianza 1 . Se generan tres categorías: (1) "deterioro" (2) "sin cambio" y (3) "mejora". Las cuales son formadas con los valores mínimos y máximos de las variables componentes formadas.

\section{RESULTADOS}

\section{Fiabilidad y validez de las variables componentes}

El análisis factorial exploratorio es factible con los datos muestrales del estudio, ya que los estadísticos de bondad de ajuste indican que las variables observables analizadas pueden explicar otras variables, como los factores formados de "Percepción de impacto a nivel personal", "A nivel familiar", "A nivel microempresarial" y "A nivel comunitario e institucional". Esto se corrobora en la Tabla 1 , ya que el determinante de la matriz de correlaciones de cada una de las variables latentes formadas es inferior a 0.10 , los coeficientes KMO (Kaiser-Meyer-Olkin) son mayores a 0.50 , así mismo, en todos los casos se rechaza la hipótesis nula de la prueba de esfericidad de Bartlett, de que la matriz de correlaciones es una matriz identidad, las cargas factoriales son superiores a 0.50 , y, la Varianza Extraída Media (AVE) es superior al 50\% en todos los casos (Merenda, 1997; Chin, 1998, Detrinidad, 2016).

Además, se comprueba la fiabilidad y validez de las variables componentes formadas mediante el análisis factorial con el coeficiente Alfa de Cronbach y la fiabilidad compuesta, que son dos medidas para evaluar la consistencia interna de factores, con la diferencia que el último tiene en cuenta la evaluación de otras variables constructo en un estudio, convirtiéndolo en un coeficiente más sofisticado (Fornell \& Larcker, 1981). En síntesis, las dos medidas indican que los factores formados presentan una buena consistencia interna, ya que son superiores a 0.70 según las recomendaciones de Cortina (1993) para el coeficiente Alpha, y Prieto \& Delgado (2010) para el coeficiente de fiabilidad compuesta.

Tabla 1. Fiabilidad y validez de las variables de impacto del microcrédito en las condiciones individuales, familiares, nivel microempresarial y comunitario.

\begin{tabular}{|c|c|c|c|c|c|c|c|}
\hline Variables & $\begin{array}{l}\text { Determinante } \\
\text { matriz de } \\
\text { correlación }\end{array}$ & KMO & $\begin{array}{l}\text { Bartlett } \\
\text { Sig. }\end{array}$ & $\begin{array}{c}\text { Cargas } \\
\text { factoriales } \\
\text { (rango) }\end{array}$ & $\begin{array}{c}\text { Varianza } \\
\text { extraída } \\
\text { AVE } \\
\end{array}$ & $\begin{array}{l}\text { Alpha } \\
\text { Cronbach }\end{array}$ & $\begin{array}{l}\text { Fiabilidad } \\
\text { compuesta }\end{array}$ \\
\hline A nivel personal & .001 & 0.844 & .000 & $0.481-0.946$ & 0.6172 & 0.893 & 0.7999 \\
\hline A nivel familiar & $1.935 \mathrm{E}-005$ & 0.819 & .000 & $0.650-0.923$ & 0.6702 & 0.935 & 0.8317 \\
\hline $\begin{array}{l}\text { A nivel micro } \\
\text { empresarial }\end{array}$ & .0138 & 0.744 & .000 & $0.606-0.836$ & 0.5927 & 0.821 & 0.7898 \\
\hline $\begin{array}{l}\text { A nivel comunitario } \\
\text { e institucional }\end{array}$ & .019 & 0.789 & .000 & $0.731-0.928$ & 0.7155 & 0.893 & 0.8555 \\
\hline
\end{tabular}




\section{Impacto del microcrédito en las condiciones de vida}

Los resultados del análisis de la percepción a nivel personal, familiar, microempresarial y comunitario de las personas socias que recibieron el crédito, diferenciando por zona, según la recodificación de las variables componentes formadas con el análisis factorial evidencia que, el $52 \%$ de las personas socias que recibieron el microcrédito en la zona urbana perciben un mejoramiento de su condición de vida a nivel personal, mientras que, en las zonas rurales, baja al $22 \%$. Por el contrario, tanto en la zona urbana como rural, un $27 \%$ de las personas socias consideran que el microcrédito ha deteriorado su condición de vida a nivel personal (Fig. 1).

Analizando a nivel de medias (Tabla 2), se encuentra una interacción significativa de la percepción de la condición de vida a nivel personal recodificada (deterioro, sin cambio, mejora) con la zona $\left[F(2.542)=32.37 ; p<.05 ; \eta^{2}\right.$ $=.11]$ en la percepción de impacto del crédito a nivel personal. Así mismo, se encuentra un efecto principal significativo de la zona y de la percepción de la condición de vida a nivel personal recodificada, $[F(1.542)=443.91$; $\left.p<.05 ; \eta^{2}=.45\right],\left[F(2.542)=1763.29 ; p<.05 ; \eta^{2}=.87\right]$, en la percepción de impacto del crédito a nivel personal, respectivamente (Tabla 3). En otras palabras, significa que existen diferencias estadísticamente significativas en las puntuaciones medias de la percepción de impacto del microcrédito a nivel personal entre las personas socio/as de las zonas rurales y urbanas, resultados que se corroboran con la comparación por pares (Tabla 4).

A nivel familiar, el 57\% de las personas socias de la zona urbana perciben una mejora de su condición de vida, mientras que, en la zona rural, un $37 \%$ percibe una mejora. En cambio, el $27 \%$ de las personas socias de las zonas rurales perciben un deterioro de las condiciones de vida familiares por el otorgamiento del crédito, mientras que, en las zonas urbanas, el 10\% tiene esa misma percepción (ver Fig. 1). Analizando las medias, se encuentra una interacción significativa de la percepción de la condición de vida a nivel familiar recodificada con la zona $[F(2.542)$ $\left.=223.42 ; p<.05 ; \eta^{2}=.45\right]$ en la percepción de impacto del crédito a nivel familiar. Así mismo, se encuentra un efecto principal significativo de la zona y de la percepción de la condición de vida a nivel familiar recodificada, $[F(1.542)$ $\left.=917.82 ; p<.05 ; \eta^{2}=.63\right],[F(2.542)=2176.66 ; p<.05 ;$ $\left.\eta^{2}=.89\right]$, en la percepción de impacto del crédito a nivel familiar, respectivamente. Esto significa que las diferencias en las puntuaciones medias de la percepción de impacto del microcrédito a nivel familiar entre las personas socio/as de las zonas rurales y urbanas son estadísticamente significativas, la comparación por pares muestra la significancia al $99 \%$ de confianza.

A nivel microempresarial, el $57 \%$ y el $54 \%$ de las personas socias de las zonas urbanas y rurales, respectivamente, perciben una mejora, mientras que, la diferencia aumenta en la percepción de deterioro de las condiciones microempresariales por el otorgamiento del crédito, en las zonas rurales es de $14 \%$ y, en las urbanas un $27 \%$. A nivel de medias, se encuentra una interacción significativa de la percepción de la condición de vida a nivel microempresarial recodificada con la zona $[F(2.542)=$ 125.76; $\left.p<.05 ; \eta^{2}=.32\right]$ en la percepción de impacto del crédito a nivel microempresarial. Así mismo, se encuentra un efecto principal significativo de la zona y de la percepción de la condición de vida a nivel microempresarial recodificada, $[F(1.542)=695.2 ; p<.05$; $\left.\eta^{2}=.56\right],\left[F(2.542)=1805.72 ; p<.05 ; \eta^{2}=.87\right]$, en la percepción de impacto del crédito a nivel microempresarial, respectivamente. Por tanto, significa que las diferencias en las puntuaciones medias encontradas en la percepción de impacto del microcrédito a nivel microempresarial entre las personas de las zonas rurales y urbanas son estadísticamente significativas, al 99\% de confianza según la comparación por pares.

Finalmente, a nivel comunitario, el $50 \%$ de las personas socias de las zonas urbanas perciben una mejora, en tanto que, en las zonas rurales, baja al $38 \%$. Situación contraria se observa, en las zonas rurales, donde la percepción de deterioro de las condiciones comunitarias incrementa (43\%) en comparación con las zonas urbanas (5\%). A nivel de medias, se encuentra una interacción significativa de la percepción de la condición de vida a nivel comunitario recodificada con la zona $[F(2.542)=17.56$;
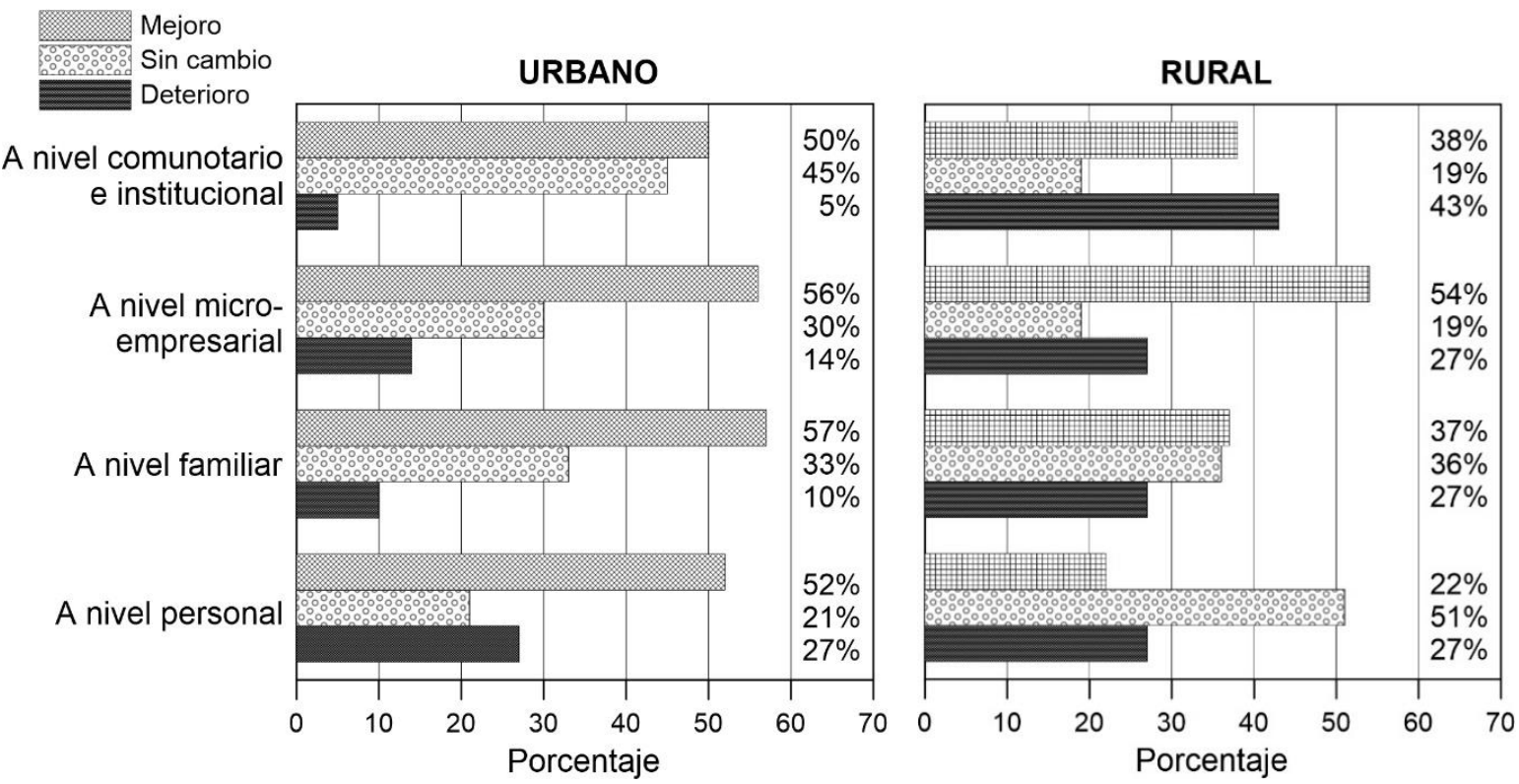

Figura 1. Condiciones de vida de socios/as que obtuvieron microcréditos en el período 2012-2017, por zona. 
Tabla 2. Puntuaciones medias de la percepción de las condiciones de vida de socios(as) por zona.

\begin{tabular}{|c|c|c|c|c|c|c|c|c|c|}
\hline \multirow{2}{*}{\multicolumn{2}{|c|}{$\begin{array}{l}\text { Condiciones de vida } \\
\text { Zona }\end{array}$}} & \multirow{2}{*}{$\begin{array}{l}\text { A nivel personal } \\
\text { Media }\end{array}$} & \multicolumn{3}{|c|}{ A nivel familiar } & \multicolumn{2}{|c|}{ A nivel microempresarial } & \multicolumn{2}{|c|}{ A nivel comunitario } \\
\hline & & & Desviación típica & Media & Desviación típica & Media & Desviación típica & Media & Desviación típica \\
\hline \multirow[t]{4}{*}{ Rural } & Deterioro & -.8873 & .2716 & -.7012 & .3316 & -.8450 & .259 & -1.2209 & .3537 \\
\hline & Sin cambio & .4193 & 1507 & -.1068 & 2158 & .2666 & 1430 & -.3011 & .0815 \\
\hline & Mejoro & 1.0709 & .2631 & .9735 & .0866 & .8808 & .2874 & 1.0614 & .4887 \\
\hline & Total & .2064 & .7500 & .1343 & .7244 & 2955 & .7804 & -.1822 & 1.0960 \\
\hline \multirow[t]{4}{*}{ Urbano } & Deterioro & -1.6731 & .3267 & -2.6364 & .4835 & -2.069 & 3919 & -2.0589 & .4516 \\
\hline & Sin cambio & -.3856 & 4681 & -.7312 & .3718 & -.8611 & .2655 & -.5099 & .3529 \\
\hline & Mejoro & .7850 & .3338 & .7259 & .3200 & .6491 & .4045 & .8932 & .3932 \\
\hline & Total & -.1272 & 1.1089 & -0828 & 1.1302 & -.1822 & 1.0753 & .1123 & .9198 \\
\hline
\end{tabular}

Tabla 3. Pruebas de los efectos inter sujetos de las condiciones de vida por zona.

\begin{tabular}{|c|c|c|c|c|c|c|c|c|c|c|c|c|}
\hline \multirow{2}{*}{$\begin{array}{l}\text { Variable dependiente } \\
\text { Origen }\end{array}$} & \multicolumn{3}{|c|}{ Nivel personal $^{1}$} & \multicolumn{3}{|c|}{ Nivel familiar $^{2}$} & \multicolumn{3}{|c|}{ Nivel microempresarial $^{3}$} & \multicolumn{3}{|c|}{ Nivel comunitario $^{4}$} \\
\hline & $\mathrm{F}$ & Sig. & $\begin{array}{c}\text { Eta al } \\
\text { cuadrado } \\
\text { parcial } \\
\end{array}$ & $\mathrm{F}$ & Sig. & $\begin{array}{c}\text { Eta al } \\
\text { cuadrado } \\
\text { parcial }\end{array}$ & $\mathrm{F}$ & Sig. & $\begin{array}{c}\text { Eta al } \\
\text { cuadrado } \\
\text { parcial }\end{array}$ & $\mathrm{F}$ & Sig. & $\begin{array}{c}\text { Eta al } \\
\text { cuadrado } \\
\text { parcial }\end{array}$ \\
\hline Modelo corregido & 985.990 & .000 & .901 & 1016.215 & .000 & .904 & 898.412 & .000 & .892 & 651.045 & .000 & .857 \\
\hline Intersección & 56.713 & .000 & .095 & 714.093 & .000 & .569 & 407.827 & .000 & .429 & 256.163 & .000 & .321 \\
\hline Zona & 443.905 & .000 & .450 & 917.815 & .000 & .629 & 695.204 & .000 & .562 & 82.879 & .000 & .133 \\
\hline Recod. condición vida & 1763.286 & .000 & .867 & 2176.661 & .000 & .889 & 1805.722 & .000 & .870 & 1250.212 & .000 & .822 \\
\hline \multirow{2}{*}{ Zona * Recod. condición vida } & 32.371 & .000 & .107 & 223.424 & .000 & .452 & 125.755 & .000 & .317 & 17.557 & .000 & .061 \\
\hline & \multicolumn{3}{|c|}{$\begin{array}{c}{ }^{1} \text { Suma de cuadrados tipo III, con } \\
\mathrm{R} \text { cuadrado }=.901 \text { ( } \mathrm{R} \text { cuadrado } \\
\text { corregida }=.900)\end{array}$} & \multicolumn{3}{|c|}{$\begin{array}{c}{ }^{2} \text { Suma de cuadrados tipo III, con } \\
\mathrm{R} \text { cuadrado }=.904 \text { (R cuadrado } \\
\text { corregida }=.903)\end{array}$} & \multicolumn{3}{|c|}{$\begin{array}{c}{ }^{3} \text { Suma de cuadrados tipo III, con } \\
\mathrm{R} \text { cuadrado }=.892 \text { ( } \mathrm{R} \text { cuadrado } \\
\text { corregida }=.891)\end{array}$} & \multicolumn{3}{|c|}{$\begin{array}{c}{ }^{4} \text { Suma de cuadrados tipo III, con } \\
\mathrm{R} \text { cuadrado }=.857 \text { ( } \mathrm{R} \text { cuadrado } \\
\text { corregida }=.856)\end{array}$} \\
\hline
\end{tabular}

*Se aplica ANOVA factorial a pesar del incumplimiento del supuesto

** Significancia bilateral al 0,05 . *** Significancia bilateral al 0,01 .

Tabla 4. Comparación por pares: condiciones de vida de socios(as) por zona.

\begin{tabular}{|c|c|c|c|c|c|c|c|c|c|c|}
\hline \multirow{2}{*}{$\begin{array}{l}\text { Variable dependiente } \\
\text { Recod. percepción } \\
\text { cond. de vida }\end{array}$} & & \multirow[b]{2}{*}{ Zona } & \multicolumn{2}{|c|}{ Nivel personal } & \multicolumn{2}{|c|}{ Nivel familiar } & \multicolumn{2}{|c|}{ Nivel microempresaria } & \multicolumn{2}{|c|}{ Nivel comunitario } \\
\hline & & & $\begin{array}{l}\text { Diferencia de } \\
\text { medias (I-J) a }\end{array}$ & Error tip. & $\begin{array}{l}\text { Diferencia de } \\
\text { medias (I-J) a }\end{array}$ & Error tip. & $\begin{array}{l}\text { Diferencia de } \\
\text { medias (I-J) a }\end{array}$ & Error tip. & $\begin{array}{l}\text { Diferencia de } \\
\text { medias (I-J) a }\end{array}$ & Error tip. \\
\hline \multirow[t]{2}{*}{ Deterioro } & Rural & Urbano & $.786^{* * * *}$ & .053 & $1.935^{* * *}$ & .068 & $1.224^{* * *}$ & .065 & $.838^{* * * *}$ & .103 \\
\hline & Urbano & Rural & $-.786^{* * * *}$ & .053 & $-1.935^{* * *}$ & .068 & $-1.224^{* * * *}$ & .065 & $-.838^{* * * *}$ & .103 \\
\hline \multirow[t]{2}{*}{ Sin cambio } & Rural & Urbano & $.805^{* * * *}$ & .048 & $.624^{* * * *}$ & .047 & $1.128^{* * * *}$ & .062 & $.209^{* * *}$ & .067 \\
\hline & Urbano & Rural & $-.805^{* * *}$ & .048 & $-.624^{* * * *}$ & .047 & $-1.128^{* * * *}$ & .062 & $-.209^{* * *}$ & .067 \\
\hline \multirow[t]{2}{*}{ Mejora } & Rural & Urbano & $.286^{* * *}$ & .052 & $.248^{* * * *}$ & .042 & $.232^{* * * *}$ & .039 & $.168^{* * *}$ & .052 \\
\hline & Urbano & Rural & $-.286^{* * *}$ & .052 & $-.248^{* * *}$ & .042 & $-.232^{* * *}$ & .039 & $-.168^{* * *}$ & .052 \\
\hline
\end{tabular}

a. Basadas en las medias marginales estimadas. Ajuste para comparaciones múltiples: Bonferroni. ** Significancia bilateral al 0,05. *** Significancia bilateral al 0,01 
$\left.p<.05 ; \eta^{2}=.06\right]$ en la percepción de impacto del crédito a nivel comunitario. Así mismo, se encuentra un efecto principal significativo de la zona y de la percepción de la condición de vida a nivel comunitario recodificada, $[F$ $\left.(1.542)=82.88 ; p<.05 ; \eta^{2}=.13\right],[F(2.542)=1250.21$; $\left.p<.05 ; \eta^{2}=.82\right]$, en la percepción de impacto del crédito a nivel comunitario, respectivamente. Esto significa que las diferencias encontradas en las puntuaciones medias de la percepción de impacto del microcrédito a nivel comunitario entre las personas de las zonas rurales y urbanas son estadísticamente significativas al $99 \%$ de confianza según la comparación por pares.

\section{DISCUSIÓN}

En este documento se evidencia la percepción de las personas socias respecto al impacto del microcrédito en sus condiciones de vida, por zonas. Así las socias y socios de la COAC Riobamba Ltda., pertenecientes a la zona urbana indican percibir en su mayoría mejoras en sus condiciones de vida, tanto a nivel personal, familiar, microempresarial y comunitario. Mientras que las personas socias de las zonas rurales en su mayoría perciben mejoras en sus condiciones de vida a nivel microempresarial, no así a nivel personal, familiar y comunitario.

Al respecto el estudio realizado por Manrique-Joya, Ramírez, \& Santos-Varón (2017) sobre el impacto de los microcréditos en la pobreza rural, dan cuenta que estos tienen impactos positivos, ya que a nivel microempresarial ha contribuido a la acumulación de capital, lo que se traduce en mejores condiciones de vida a mediano y largo plazo. En esta misma lógica, el estudio realizado por Maldonado Román \& Armijos Tandazo (2017), demuestra que los microcréditos aportan a la creación de emprendimientos, constituyéndose en la principal herramienta que permite cumplir con las expectativas empresariales que a largo plazo aportan al mejoramiento de sus condiciones de vida. De igual manera, el estudio realizado por Carvajal-Salgado \& Espinoza-Párraga (2020) demuestra que el microcrédito al mitigar la pobreza patrimonial y alimentaria, a nivel familiar contribuye al aumento de su calidad de vida. Así también, la investigación de Yánez Rodríguez (2012) respecto al impacto del microcrédito en zonas productivas, evidencia que una efectiva gestión del microcrédito trae consigo cambios positivos dentro de la comunidad, ya que genera empleo, impulsado el sector microempresarial, que conlleva a la mejora de las condiciones de vida de la comunidad. Finalmente, el estudio Orozco-Aguilar (2016) muestra que el microcrédito tiene impacto a nivel personal, sobre todo en las mujeres, quienes, al manejar los recursos, han adquirido confianza en sí mismas, situación que les ayuda a poder tomar decisiones para ellas y su hogar.

Por otra parte, los resultados de esta investigación demuestran también que los microcréditos son percibidos también como instrumentos que han generado deterioro de las condiciones de vida de las personas socias de la COAC Riobamba Ltda., así, un porcentaje de personas socias de la cooperativa, pertenecientes a la zonas urbanas y rurales perciben que los microcréditos han generado deterioro de sus condiciones de vida, tanto a nivel personal, familiar, microempresarial y comunitario. En promedio, la percepción de deterioro de las condiciones de vida es mayor en socios y socias de la zona urbanas que de las zonas rurales. Esto tiene sentido en el hecho de que un mal manejo de un microcrédito puede afectar más significativamente en las zonas urbanas, ya que dependen exclusivamente del recurso económico (monetario) para acceder a bienes y servicios necesarios para sustento personal, familiar y microempresarial, que, en las zonas rurales, en donde de alguna forma hacen uso del recurso tierra y las prácticas del intercambio para conseguir los bienes y servicios para el sustento de sus condiciones de vida, por esta razón se sustentan los efectos significativos de la percepción de impacto de los microcréditos en las condiciones de vida por zona. En este contexto, el trabajo de Gómez-Gil (2019) evidencia que el microcrédito presenta problemas instrumentales y estructurales que profundizan la pobreza y la desigualdad, por tanto, se deterioran las condiciones de vida a todo nivel.

Del análisis de las diferencias significativas, los resultados de esta investigación muestran que los socios de las zonas rurales tienen una mayor percepción de "mejora" en las puntuaciones medias del impacto del crédito tanto a nivel personal, familiar, microempresarial y comunitario en comparación con las personas socias de las zonas urbanas, por ello, la comparación por pares de la condición de "mejora" Rural-Urbano da diferencias positivas tanto a nivel personal, familiar, microempresarial y comunitario, lo contrario se encuentra en la comparación de "mejora" Urbano-Rural en todos los niveles de la percepción de impacto del microcrédito. En contraste con esta información, el estudio de Estrada \& Hernández-Rubio (2019) demuestra que el impacto positivo de los microcréditos es mayor en las zonas urbanas, donde se disminuye el hecho de continuar en situaciones de pobrezas, sin embargo, no se encuentra efectos estadísticamente significativos en la zona rural.

\section{CONCLUSIONES}

Se debe expresar que la evaluación de impacto del microcrédito permite identificar cambios en la vida de los individuos que accedieron a este. Desde esta perspectiva, los resultados de esta investigación muestran la percepción de las personas socias de la COAC Riobamba Ltda., respecto al impacto del microcrédito, determinando que para la mayoría este se constituye en una alternativa que aporta al mejoramiento de las condiciones de vida a nivel personal como familiar, microempresarial y comunitario, sobre todo en la zona urbana. Esta percepción, según se expresa en marco teórico de esta investigación, se debe a que, el microcrédito potencia y fortalece iniciativas productivas que les permitió generar autoempleo y recursos que facilitan el acceso a educación, salud, vivienda. Cabe recalcar que la percepción no es igual para todas las personas asociadas, ya que, en su mayoría, las socio/as de las zonas rurales perciben una mejora a nivel familiar y microempresarial, más no a nivel personal y comunitario.

Por otro parte, los resultados ponen de manifiesto que, algunos socios y socias consideran que el microcrédito también deterioró las condiciones de vida tanto a nivel personal, familiar, microempresarial y comunitario; aunque esta percepción es mayor en las zonas urbanas que en las rurales. Esto concuerda con la revisión teórica, al 
considerar que un uso inadecuado de los recursos del microcrédito puede endeudar a quienes acceden al mismo, generando deterioro de las condiciones de vida tanto a nivel personal, familiar, microempresarial y comunitaria.

En términos generales, el desarrollo del presente estudio permite concluir que, el microcrédito como alternativa financiera es un instrumento idóneo para mejorar las condiciones de vida de los socios y socias de la COAC Riobamba Ltda., y la de sus familias, redundando a mediano y largo plazo en la mejora de las condiciones de vida de la comunidad. Por tanto, el microcrédito se constituye en potenciador del desarrollo local. Resultado que comprueba la hipótesis planteada, pues la percepción de las personas socias confirman que el acceder a un microcrédito se constituye en una oportunidad para emprender, generando autoempleo $\mathrm{y}$, con ello, acceder a ingresos que permitieron mejorar sus condiciones de vida a nivel personal, familiar, microempresarial y comunitaria. Siempre que su uso sea adecuado, es decir, invertido en actividades productivas que mejoren sus ingresos, con lo cual, conlleva a la mejora de las condiciones de vida.

\section{REFERENCIAS}

Álava Atiencie, N. G. (2019). Sostenibilidad de organizaciones agroecológicas que apoyan al fomento de la economía popular y solidaria en la provincia del Azuay. Madrid, España: Universidad de Complutense, Tesis doctoral, $487 \mathrm{pp}$. Obtenido de https://core.ac.uk/download/pdf/287737047.pdf

Cairó I Céspedes, G., \& Gómez Gonzáles, L. K. (2015). $\mathrm{El}$ enfoque financiero vs. el enfoque social del microcrédito. Un análisis comparativo mundial. Revista de Estudios Cooperativos, 118(1), 31-59. https://doi.org/10.5209/rev_REVE.2015.n118.49062

Carvajal-Salgado, A., \& Espinoza-Párraga, L. (2020). Microcréditos ecuatorianos: incentivo a la reducción de la pobreza y mejora del ingreso familiar. VínculosESPE, 5(3), 51-65. doi:

10.24133/vinculosespe.v5i3.1671

Casas, F. (2004). De afrontar problemas sociales a promover la calidad de vida. Revista de Historia de la Psicología, 25(4), 305-322.

Cátedra Iberoamericana. (2006). Impacto social y económico del microcrédito. Universität de les Illes Balears. Obtenido de

http://fci.uib.es/Servicios/libros/investigacion/Bukstein /XVI--Impacto-social-y-economico-delmicrocredito.cid217676

Chin, W. W. (1998). The partial least squares approach to structural equation modeling. En G. A. Marcoulides (Ed), Modern Methods for Business Research (pp. 295336). New Jersey, USA: Lawrence Erlbaum Associates, Publisher.

Cisneros-Martínez, M., \& Garcés- Venegas, A. (2021). Microcrédito: un perfil a socios de los bancos comunales del cantón Cevallos. Boletín de Coyuntura, n.29, 20-27.

Cortina, J. M. (1993). What is coefficient Alpha? An examination of theory and applications. Journal of
Applied Psychology, 78(1), 98-104. https://doi.org/10.1037/0021-9010.78.1.98

Detrinidad, E. (2016). Análisis factorial exploratorio y confirmatorio aplicado al modelo de secularización propuesto por Inglehart-Norris. Periodo 2010-2014. (Estudio de caso España, Estados Unidos, Alemania, Holanda) WSV. Tesis de Maestría, Universidad de Granada. Obtenido de

https://www.researchgate.net/publication/327393652_ Analisis_Factorial

_Exploratorio_y_Confirmatorio_aplicado_al_modelo_ de_secularizacion_propuesto_por_InglehartNorris_Periodo_2010-2014_Estudio_de_caso_ Espana_Estados_Unidos_Alemania_Holanda_WSV

Estrada, D., \& Hernández-Rubio, A. (2019). Situación actual e impacto del microcrédito en Colombia. 261 pp. ASOMICROFINANZAS, Banco de la Republica Colombia. Obtenido de https://repositorio.banrep.gov.co/bitstream/handle/20.5 00.12134/9723/LBR_2019-07.pdf

Fornell, C., \& Larcker, D. (1981). Evaluating structural equation models with unobservable variables and measurement error. Journal of Marketing Research, n. 18, 39-50.

García, L. (2011). Evaluación de impacto aplicada a un proyecto de mercadeo social. Finanzas y Política Económica, 3(1), 39-57.

Gómez-Acebo, M. (2018). Análisis de las metodologías de evaluación de impacto de los programas de microcréditos en América Latina. Trabajo Fin de Grado, Universidad Pontificia Comillas. Obtenido de https://repositorio.comillas.edu/xmlui/bitstream/handle/ 11531/22173/TFG-

Gomez\%20Acebo\%20Lopez\%2c\%20Monica.pdf?sequ ence $=1 \&$ isAllowed $=\mathrm{y}$

Gómez-Gil, C. (2019). El fenómeno de los microcréditos en la cooperación mundial: límites y controversias. Universidad de Alicante, Ensayo, n.9, 6-20. Obtenido de https://core.ac.uk/download/pdf/224796365.pdf

Gómez-Lavín Fernández, M. (2015). Microfinanzas y las escuelas de pensamiento: Análisis del caso Banco Compartamos. Madrid. Trabajo Fin de Grado, Universidad Pontificia Comillas. Obtenido de https://repositorio.comillas.edu/jspui/bitstream/11531/4 450/1/TFG001240.pdf

Lema, P., Erazo, J., \& Narváez, C. (2019). El talento humano, factor clave para la gestión organizacional en Instituciones de intermediación financiera. Revista Arbitrada Interdisciplinaria Koinonia, 4(1), 349-375. doi: http://dx.doi.org/10.35381/r.k.v4i1.461

Maldonado Román, M. B., \& Armijos Tandazo, L. A. (2017). Los microcréditos y su incidencia en el crecimiento económico de las Mipymes. Revista Ser Academia, 7(1), 19-24.

Martínez-Centanaro, A. (2017). Efectos del microcrédito sobre las condiciones de vida de la población vulnerable del municipio de Sincelejo programa si emprende. Maestría en Desarrollo Social, Universidad del Norte. Obtenido de https://manglar.uninorte.edu.co/bitstream/ handle/10584/8747/138710.pdf?sequence=1\&isAllowe $\mathrm{d}=\mathrm{y}$ 
Méndez, C., \& Rondón, M. A. (2012). Introducción al análisis factorial exploratorio. Revista Colombiana de Psiquiatría, 41(1), 197-207.

Merenda, P. (1997). A guide to the proper use of factor analysis in the conduct and reporting of research: Pitfalls to avoid. Measurement and Evaluation in Counseling and Development, 30(3), 156-164. https://doi.org/10.1080/07481756.1997.12068936

Naciones Unidas. (1997). Declaración y plan de acción de la cumbre sobre el microcrédito. Washington, D.C. Obtenido de https://undocs.org/pdf?symbol=es/A/52/113

Prieto, G., \& Delgado, A. R. (2010). Fiabilidad y validez. Papeles del Psicólogo, 31(1), 67-74.

Rodríguez-Garcés, C. (2008). Impacto de las microfinanzas: resultados de algunos estudios, Énfasis en el sector financiero. Revista Ciencias Estratégicas, 16(20), 281-298.
Rué Cabré, E. (2006). Desarrollo y capacidades: aplicación al microcrédito desde una perspectiva de género. Revista CIDOB d'Afers Internacionals, n.60, 161-184.

Sanhueza, P. (2019). Impacto de las microfinanzas en la microempresa local. Dimensión Empresarial, 17(2), 5-19. https://doi.org/10.15665/dem.v17i2.1933

Sen, A. (2000). Desarrollo y libertad. (1a. ed. en español). Buenos Aires, Argentina: Editorial Planeta. Obtenido de https://www.palermo.edu/Archivos_ content/2015/derecho/pobreza_multidimensional/ bibliografía/ Sesion1_doc1.pdf 